\title{
Rotating molecular outflows: the young T Tauri star in CB $26^{\star}, \star \star$
}

\author{
R. Launhardt ${ }^{1}$, Ya. Pavlyuchenkov ${ }^{1,2}$, F. Gueth ${ }^{3}$, X. Chen ${ }^{1}$, A. Dutrey ${ }^{4,5}$, S. Guilloteau ${ }^{4,5}$, Th. Henning ${ }^{1}$, V. Piétu ${ }^{3}$, \\ K. Schreyer ${ }^{6}$, and D. Semenov ${ }^{1}$
}

1 Max-Planck-Institut für Astronomie, Königstuhl 17, 69117 Heidelberg, Germany e-mail: rl@mpia.de

2 Institute of Astronomy, Russian Academy of Sciences, Pyatnitskaya 48, Moscow 109117, Russia

3 IRAM, 300 rue de la piscine, 38406 Saint-Martin-d'Hères, France

${ }_{5}^{4}$ Université Bordeaux 1, Laboratoire d'Astrophysique de Bordeaux (LAB), France

5 CNRS/INSU - UMR5804, BP 89, 33270 Floirac, France

6 Astrophysikalisches Institut und Universitäts-Sternwarte, Schillergässchen 2-3, 07745 Jena, Germany

Received 20 August 2008 / Accepted 18 November 2008

\begin{abstract}
Context. The disk-outflow connection is thought to play a key role in extracting excess angular momentum from a forming proto-star. Although jet rotation has been observed in a few objects, no rotation of molecular outflows has been unambiguously reported so far. Aims. We report new millimeter-interferometric observations of the edge-on T Tauri star - disk system in the isolated Bok globule CB 26. The aim of these observations was to study the disk-outflow relation in this 1 Myr old low-mass young stellar object. Methods. The IRAM PdBI array was used to observe ${ }^{12} \mathrm{CO}(2-1)$ at $1.3 \mathrm{~mm}$ in two configurations, resulting in spectral line maps with $1.5^{\prime \prime}$ resolution. We use an empirical parameterized steady-state outflow model combined with 2-D line radiative transfer calculations and $\chi^{2}$-minimization in parameter space to derive a best-fit model and constrain parameters of the outflow.

Results. The data reveal a previously undiscovered collimated bipolar molecular outflow of total length $\approx 2000$ AU, escaping perpendicular to the plane of the disk. We find peculiar kinematic signatures that suggest that the outflow is rotating with the same orientation as the disk. However, we could not ultimately exclude jet precession or two misaligned flows as possible origins of the observed peculiar velocity field. There is indirect indication that the embedded driving source is a binary system, which, together with the youth of the source, could provide a clue to the observed kinematic features of the outflow.

Conclusions. CB 26 is so far the most promising source in which to study the rotation of a molecular outflow. Assuming that the outflow is rotating, we compute and compare masses, mass flux, angular momenta, and angular momentum flux of the disk and outflow and derive disk dispersal timescales of $0.5 \ldots 1 \mathrm{Myr}$, comparable to the age of the system.
\end{abstract}

Key words. circumstellar matter - ISM: jets and outflows - stars: pre-main sequence - planetary systems: protoplanetary disks ISM: molecules

\section{Introduction}

A comprehensive understanding of the complex relations between a collapsing molecular cloud core, the forming young (proto-)star, its circumstellar disk, and the expelled bipolar jets is a necessary prerequisite to understand the formation of stars and planetary systems. In particular, the initial amount, evolution, and re-distribution of angular momentum appear to be key factors in many of the processes which determine the protostellar fragmentation, the final stellar mass(es), and the existence and morphology of planetary systems.

Rotation of protostellar jets and molecular outflows about their symmetry axis is believed to be a key mechanism to remove

\footnotetext{
* Based on observations carried out with the IRAM Plateau de Bure Interferometer. IRAM is supported by INSU/CNRS (France), MPG (Germany) and IGN (Spain). Also based on observations collected at the Centro Astronómico Hispano Alemán (CAHA) at Calar Alto, operated jointly by the Max-Planck Institut für Astronomie and the Instituto de Astrofísica de Andalucía (CSIC).

$\star \star$ A complete set of channel maps is available in electronic form at the CDS via anonymous ftp to cdsarc.u-strasbg.fr $(130.79 .128 .5)$ or via

http://cdsweb.u-strasbg.fr/cgi-bin/qcat?]/A+A/494/147.
}

angular momentum from forming protostars and, thus, at least partially, to solve the angular momentum problem in star formation (e.g., Ray et al. 2007). As a consequence, many groups have searched for rotation signatures in jets and outflows. Indeed, during the past few years, radial velocity ${ }^{1}$ gradients have been observed across a number of protostellar jets using highresolution HST spectra (e.g., Bacciotti et al. 2002, 2003; Coffey et al. 2004; Woitas et al. 2005; Coffey et al. 2007). These were interpreted as jet rotation tracing the helicoidal structure of the magnetic field in accretion-ejection structures. As these observations are very difficult and the rotation signatures are weak, other groups have also searched for rotation signatures in molecular outflows. Pety et al. (2006) searched, but did not detect outflow rotation in HH 30. In HH 211, Lee et al. (2007) reported radial velocity gradients across $\mathrm{SiO}$ knots which they interpret as jet rotation. However, recent PdBI observations could not confirm this claim (Gueth et al. in prep.). In HH 212, Codella et al. (2007)

\footnotetext{
1 Throughout this paper, we use the term "radial velocity" when we refer to the line-of-sight velocity in the coordinate system of the observer. For the radial component of the gas motion in the coordinate system of the source (i.e., gas moving radially away from the source), we use the term "radial outflow velocity".
} 


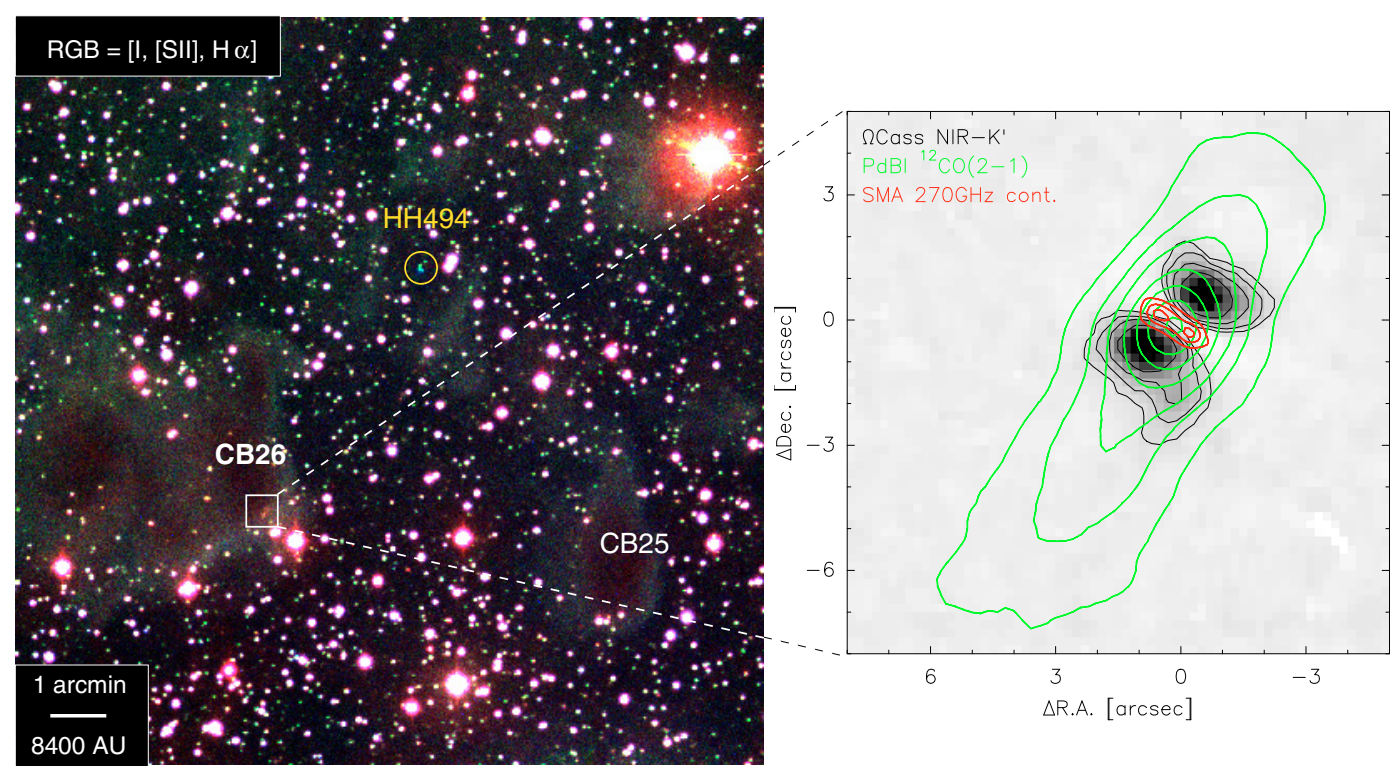

Fig. 1. Overview of the CB 26 region. The optical true-color image (left) is based on wide-field H $\alpha$ (blue), [SII] (green), and $I$-band (red) frames (Stecklum et al. 2004). The globules CB 25 and CB 26 as well as Herbig-Haro object HH 494 are marked in the image. The zoom panel (right) shows a NIR $K$-band image of the bipolar reflection nebula (grey-scale, 0 '. 6 resolution), overlaid with contours of the SMA $1.1 \mathrm{~mm}$ dust continuum emission from the disk (red, contours at 20,38, and $55 \mathrm{mJy} / \mathrm{beam})$ and the integrated ${ }^{12} \mathrm{CO}(2-1)$ emission $\left(0.6\right.$ to $\left.12.5 \mathrm{~km} \mathrm{~s}{ }^{-1}\right)$ from the bipolar molecular outflow (green, contours at $0.5,1,2,1.9,2.9, \ldots$ Jy/beam $\mathrm{km} \mathrm{s}^{-1}$ ). Beam sizes are shown in Figs. 2 and 4 . The reference position is $\alpha_{2000}=04^{\mathrm{h}} 59^{\mathrm{m}} 50.74^{\mathrm{s}}, \delta_{2000}=52^{\circ} 04^{\prime} 43.80^{\prime \prime}$.

did not find signatures of jet rotation in $\mathrm{SiO}(2-1)$ and $\mathrm{SiO}(5-4)$, but Lee et al. (2008) detected velocity gradients across the tips of two bow shocks in the $\mathrm{SiO}(8-7)$ line which they interpret as jet rotation. A recent study of $\mathrm{H}_{2}$ emission in $\mathrm{HH} 26$ and $\mathrm{HH} 72$ by Chrysostomou et al. (2008) also seems to indicate signatures of molecular jet rotation in two other embedded Class I sources.

In order to study the chemical composition, kinematics, and evolution of protoplanetary disks, we started the CID project ("Chemistry In Disks"), a molecular line survey with the IRAM Plateau de Bure Interferometer (PdBI) of well-known disks such as those surrounding LkCa 15, MWC 480, or DM Tau (Dutrey et al. 2007). Our main goal is to map, at high angular resolution, various molecular lines with enough sensitivity to derive molecular abundance variations versus radius. For the youngest source in our sample, CB 26, we also mapped the molecular outflow with the goal of studying the disk - outflow relation.

CB 26 (L 1439) is a small cometary-shaped Bok globule located $\approx 10^{\circ}$ north of the Taurus-Auriga dark cloud, at a distance of $\approx 140 \mathrm{pc}$. A dense core with signatures of star formation is located at the south-west rim of the globule (Launhardt \& Henning 1997). OVRO observations of the mm dust continuum emission and of the ${ }^{13} \mathrm{CO}(1-0)$ line have revealed a nearly edge-on circumstellar disk of radius 200 AU with Keplerian rotation (Launhardt \& Sargent 2001), surrounding a very young (obscured) low-mass T Tauri star. It is associated with a small bipolar near-infrared (NIR) nebula bisected by a dark extinction lane at the position and orientation of the edge-on disk (Stecklum et al. 2004). The source is surrounded by an optically thin asymmetric envelope with a well-ordered magnetic field directed along PA $\sim 25^{\circ}$ (Henning et al. 2001). Furthermore, a HerbigHaro object ( $\mathrm{HH} 494)$ was identified by $\mathrm{H} \alpha$ and $\mathrm{S}[\mathrm{II}]$ narrowband imaging, 6.15 arcmin northwest of $\mathrm{CB} 26$ at $\mathrm{PA}=145^{\circ}$ (Stecklum et al. 2004). The HH object is thus perfectly aligned with the symmetry axis of the disk and the bipolar nebula.

Here, we present new interferometric molecular line data of CB 26 and report the discovery of a small, well-collimated bipolar molecular outflow. The outflow has a peculiar velocity structure that suggests it is rotating with the same orientation as the disk.

\section{Observations and data reduction}

Observations of CB 26 were carried out with the IRAM PdBI in November (D configuration with 5 antennas) and December 2005 (C configuration with 6 antennas). The two array configurations provided baseline lengths in the range $16-175 \mathrm{~m}$. The phase center was at $\alpha_{2000}=04^{\mathrm{h}} 59^{\mathrm{m}} 50.74^{\mathrm{s}}, \delta_{2000}=$ $52^{\circ} 04^{\prime} 43.80^{\prime \prime}$. Two receivers were used simultaneously and tuned single side-band (SSB) to the $\mathrm{HCO}^{+}(1-0)$ line at $89.188526 \mathrm{GHz}$ and the ${ }^{12} \mathrm{CO}(2-1)$ line at $230.537984 \mathrm{GHz}$, respectively, adopting the systemic velocity ${ }^{2}$ of $\mathrm{CB} 26, v_{\mathrm{LSR}}=$ $5.5 \mathrm{~km} \mathrm{~s}^{-1}$. In this paper, we present only the ${ }^{12} \mathrm{CO}(2-1)$ data on the molecular outflow. A more detailed analysis of the disk structure, that includes other molecular line data, will be presented in a forthcoming paper.

The quasar $0355+508$ served as the phase calibrator to determine the time-dependent complex antenna gains. The correlator bandpass was calibrated on 3 C 454.3 and 3 C 273 , and the absolute flux density scale was derived from observations of MWC 349. The flux calibration uncertainty is estimated to be $\leq 20 \%$. The primary beam size at $1.3 \mathrm{~mm}$ was $22^{\prime \prime}$. The SSB system temperature at $230 \mathrm{GHz}$ was in the range $250-450 \mathrm{~K}$. Total bandwidth and effective channel spacing for the ${ }^{12} \mathrm{CO}(2-1)$ observations were $12 \mathrm{MHz}\left(16 \mathrm{~km} \mathrm{~s}^{-1}\right)$ and $32 \mathrm{kHz}\left(0.25 \mathrm{~km} \mathrm{~s}^{-1}\right)$, respectively. Zero-spacing data were obtained in September 2006 with the IRAM $30 \mathrm{~m}$ telescope at Pico Veleta in Spain. They were combined with the interferometric data to help the deconvolution. However, the central velocity channels between 4.75 and $6.75 \mathrm{~km} \mathrm{~s}^{-1}$, which are completely dominated by the extended envelope, could not be well-restored and were therefore masked out for the subsequent analysis (Fig. 2).

\footnotetext{
2 All radial velocity values in this paper refer to the Local Standard of Rest (LSR).
} 

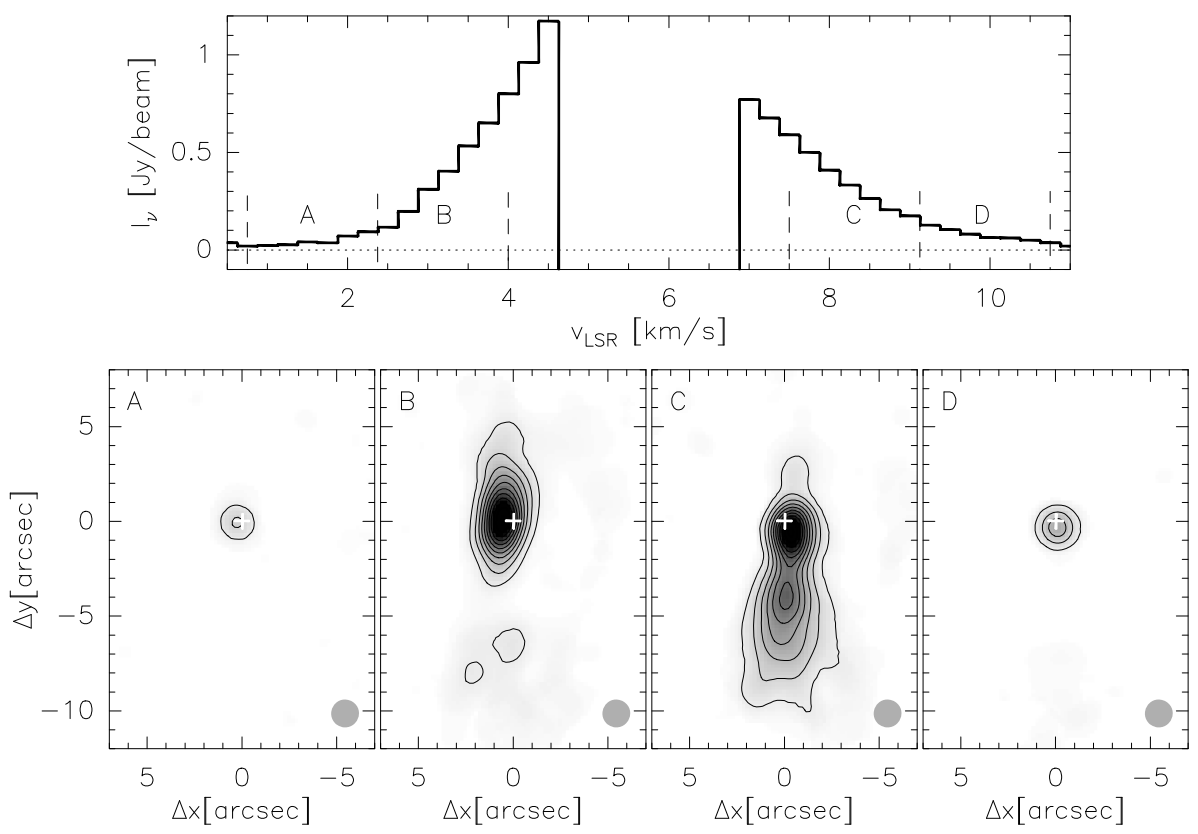

Fig. 2. ${ }^{12} \mathrm{CO}(2-1)$ spectrum and binned channel maps of CB 26. Top panel: average ${ }^{12} \mathrm{CO}(2-1)$ spectrum of the central $\pm 2{ }^{\prime \prime}$. The central velocity channels between 4.75 and $6.75 \mathrm{~km} \mathrm{~s}^{-1}$ were masked out because they are dominated by the extended envelope. The integration ranges for the four binned channel maps (bottom panels) are indicated by vertical dashed lines and marked $\mathrm{A}, \mathrm{B}, \mathrm{C}$, and D. Bottom panels: binned ${ }^{12} \mathrm{CO}(2-1)$ channel maps of $\mathrm{CB} 26$, rotated by $30^{\circ}$, with integration ranges indicated by dashed lines in the top panel. Contour levels start at $0.15 \mathrm{Jy} / \mathrm{beam} \mathrm{km} \mathrm{s}{ }^{-1}$. The white cross marks the center of the disk. The ${ }^{12} \mathrm{CO}$ synthesized beam size is indicated as grey ellipses in the lower left corners. The two outer maps show the high-velocity Keplerian wings of the inner disk. The two inner maps are dominated by the blue (upwards) and red (downwards) outflow lobes, respectively.
The data were calibrated and imaged using the GILDAS ${ }^{3}$ software. Channel maps were obtained with natural $u v$-weighting, resulting in an effective angular resolution of $1.47^{\prime \prime}$ (synthesized beam FWHM). The rms in the channel maps is $20 \mathrm{mJy} / \mathrm{beam}$.

New NIRK' -band images at $0 .{ }^{\prime} 6$ seeing and with $0 .{ }^{\prime} 2$ pixel scale were obtained in January 2008 with the Omega Cass camera (Lenzen et al. 1998) at the $3.5 \mathrm{~m}$ telescope of the Centro Astronómico Hispano Alemán (CAHA) at Calar Alto in Spain. These data are used here as complementary information to illustrate the morphology of the bipolar reflection nebula with respect to the edge-on circumstellar disk (Fig. 1). Additional SMA interferometric data at $270 \mathrm{GHz}$ to which we refer here (see also Fig. 1) will be presented in a forthcoming paper (Launhardt et al., in prep.).

\section{The ${ }^{12} \mathrm{CO}(2-1)$ maps}

The ${ }^{12} \mathrm{CO}(2-1)$ channel maps and integrated intensity maps of CB 26 reveal a small, well-collimated, slightly asymmetric bipolar molecular outflow oriented perpendicular to the plane of the disk at PA $=147 \pm 3^{\circ}$. This is illustrated in Fig. 2, which shows an integrated spectrum together with a set of binned channel maps. A complete set of channel maps is shown in Fig. 3, which is available at the CDS.

The NW lobe of the outflow is pointing towards us (blue), the SE lobe is pointing away. This is consistent with the Doppler shift of the Herbig-Haro object $\mathrm{HH} 494$, located 6.15 arcmin northwest of CB 26 on the axis of the molecular outflow (Fig. 1; Stecklum et al. 2004). At the central position, emission from the disk and outflow are superimposed. The total projected length of the bipolar molecular outflow is $14^{\prime \prime}$, which corresponds to $\approx 2000$ AU at $140 \mathrm{pc}$. However, the separation between the CB 26 disk and $\mathrm{HH} 494$ of $\approx 0.25 \mathrm{pc}$ suggests that the total extent of the outflow is much larger.

Both the 1st moment map of the ${ }^{12} \mathrm{CO}(2-1)$ emission (Fig. 4) and the position-velocity diagrams (Fig. 5) indicate the presence of a systematic velocity gradient perpendicular to the flow axis along the entire outflow. The orientation of this

${ }^{3}$ http://wWw.iram.fr/IRAMFR/GILDAS lateral velocity gradient is the same as that in the disk. The central position-velocity diagram across the disk shows the classical Keplerian velocity pattern (Fig. 5; cf. Launhardt \& Sargent 2001). The position-velocity diagrams at $+3^{\prime \prime}$ and $-4^{\prime \prime}$ away from the disk show clearly that the $\mathrm{CO}$ emission from the southwest side of the outflow axis (negative $\Delta x$ ) is red-shifted with respect to that from the north-east side (positive $\Delta x$ ) by about $2 \mathrm{~km} \mathrm{~s}^{-1}$. The amplitude of this lateral velocity gradient is comparable to that of the longitudinal velocity shift due to the bipolarity of the outflow. Although this is probably a coincidence due to the high inclination angle with respect to the line of sight (see Sect. 4.1), it has helped to reveal these kinematic features in the first place (see also discussion in Sect. 6.2). The observed radial velocity field of CB 26 is very suggestive of a rotating outflow. To test this hypothesis and to derive more quantitative results, we develop in the following section an empirical model of the disk-outflow system and compare it to the full ${ }^{12} \mathrm{CO}(2-1)$ data cube.

\section{Modeling disk and outflow}

In order to reproduce the observed ${ }^{12} \mathrm{CO}(2-1)$ maps of $\mathrm{CB} 26$, models of both the disk and the outflow are required. In this paper, we use a fixed disk model that was derived from dust continuum observations and develop an empirical model for the outflow. We then compute synthetic CO maps for the combined disk+outflow system and compare them to the observations (on the $T_{\mathrm{mb}}$ scale). Note that in the following we use two different notations for the radius vector: " $R$ " refers to cylindrical coordinates, while " $r$ " refers to spherical coordinates (see Fig. 7).

\subsection{Disk model}

For the disk, we use the best-fit empirical model derived from multi-wavelength continuum observations of CB 26 (Sauter et al., in prep.). The basis of this model is a Keplerian disk with the following density distribution:

$\rho_{\text {disk }}=\rho_{0}\left(\frac{R_{0}}{R}\right)^{\alpha_{\rho}} \exp \left[-\frac{1}{2}\left(\frac{z}{h(R)}\right)^{2}\right]$ with $h(R)=h_{0}\left(\frac{R}{R_{0}}\right)^{\alpha_{h}}$ 


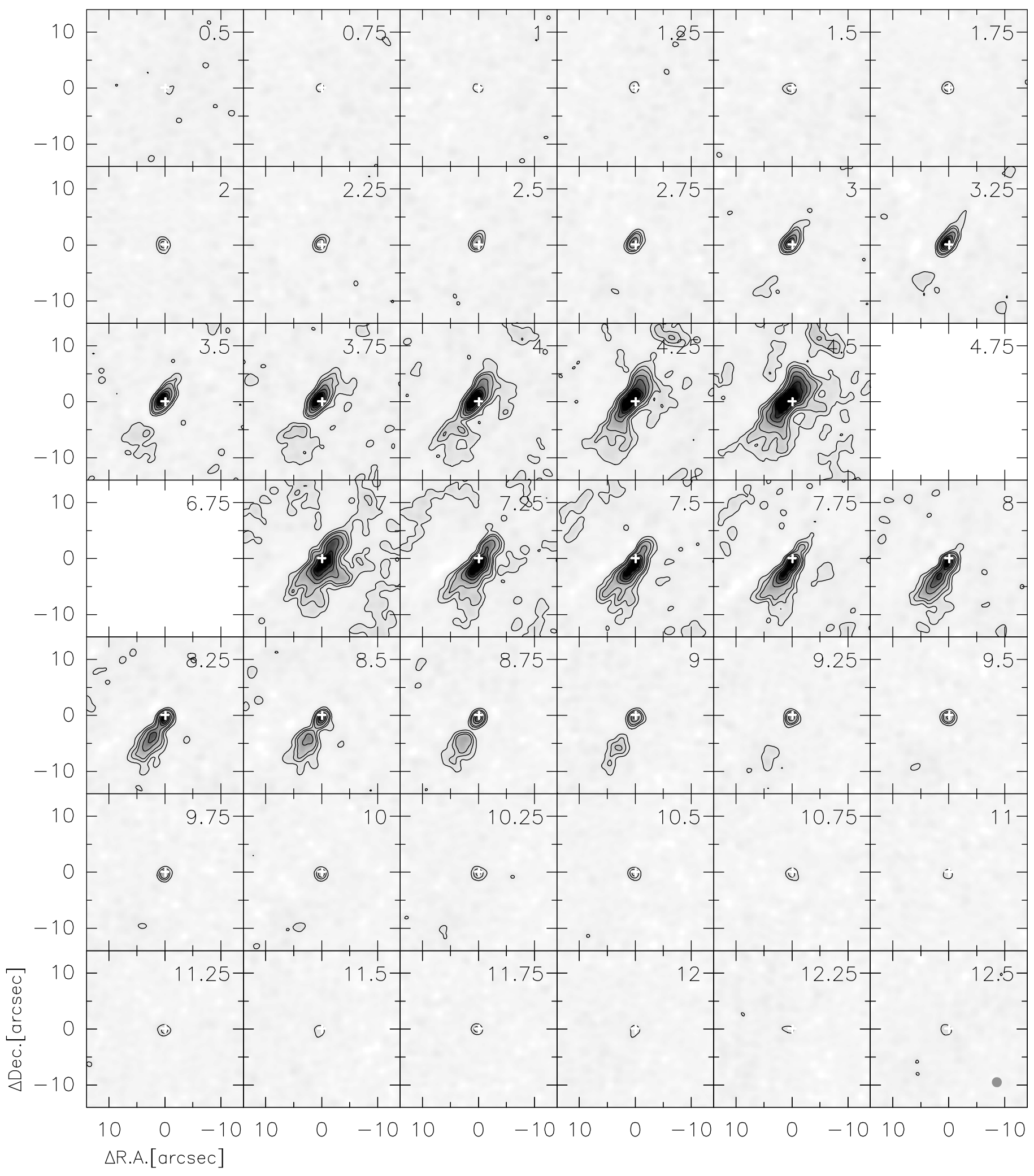

Fig. 3. ${ }^{12} \mathrm{CO}(2-1)$ channel maps of $\mathrm{CB} 26$. Contour levels start at $60 \mathrm{mJy} / \mathrm{beam}(3 \sigma \mathrm{rms})$. The white cross marks the center of the disk. The reference position is $\alpha_{2000}=04^{\mathrm{h}} 59^{\mathrm{m}} 50.74^{\mathrm{s}}, \delta_{2000}=52^{\circ} 04^{\prime} 43.80^{\prime \prime}$. The ${ }^{12} \mathrm{CO}$ synthesized beam size is indicated as the grey ellipse in the lower right corner of the last channel map. The mean $v_{\mathrm{LSR}}$ of each channel (in $\mathrm{km} \mathrm{s}^{-1}$ ) is indicated in the top right corner of the maps. The central velocity channels between 4.75 and $6.75 \mathrm{~km} \mathrm{~s}^{-1}$ were masked out because they are dominated by the extended envelope.

Here, $R$ and $z$ are the cylindrical coordinates and $R_{0}=100 \mathrm{AU}$. Furthermore, the model includes an optically thin extended envelope to account for the scattered light observed at NIR wavelengths. The disk is assumed to be passive and is heated by the central star only. Values of the free parameters $\left(\rho_{0}, \alpha_{\rho}, h_{0}\right.$, and $\alpha_{h}$ ) were derived by radiative transfer simulations with the MC3D code (Wolf et al. 2003) to fit the observed SED and the spatially resolved maps at JHKNIR-bands as well as at three 

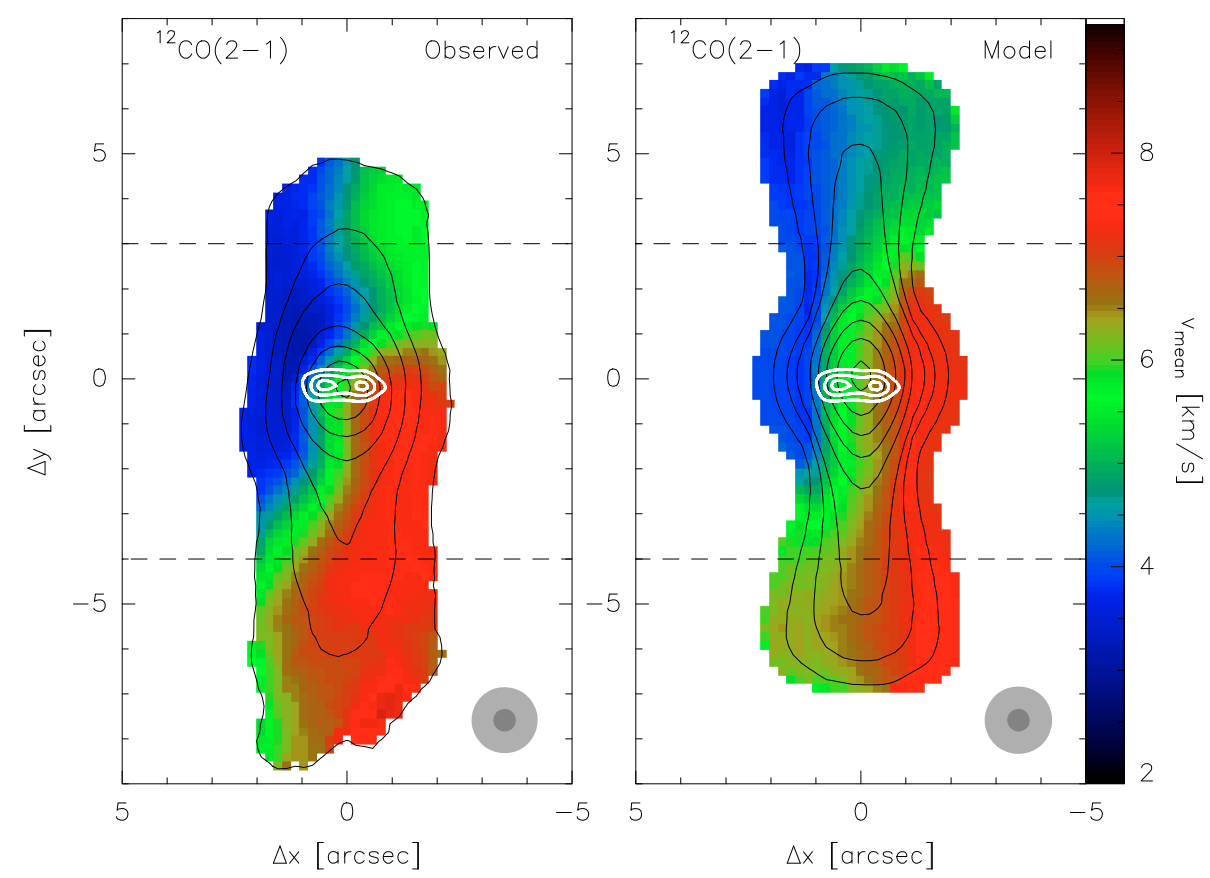

Fig. 4. ${ }^{12} \mathrm{CO}(2-1)$ integrated intensity maps (contours) and mean velocity field (1st moment map, color) of $\mathrm{CB} 26$, rotated by $30^{\circ}$. White contours show the $1.1 \mathrm{~mm}$ dust continuum emission from the disk as observed with the SMA (contour levels same as Fig. 1). The ${ }^{12} \mathrm{CO}$ synthesized beam size is shown as the large grey ellipse. The smaller and darker ellipse shows the $1.1 \mathrm{~mm}$ continuum beam. Left panel: observations. Right panel: best-fit model for ${ }^{12} \mathrm{CO}(2-1)$. Dashed lines refer to the $y$-coordinate of the position-velocity diagrams shown in Fig. 5.
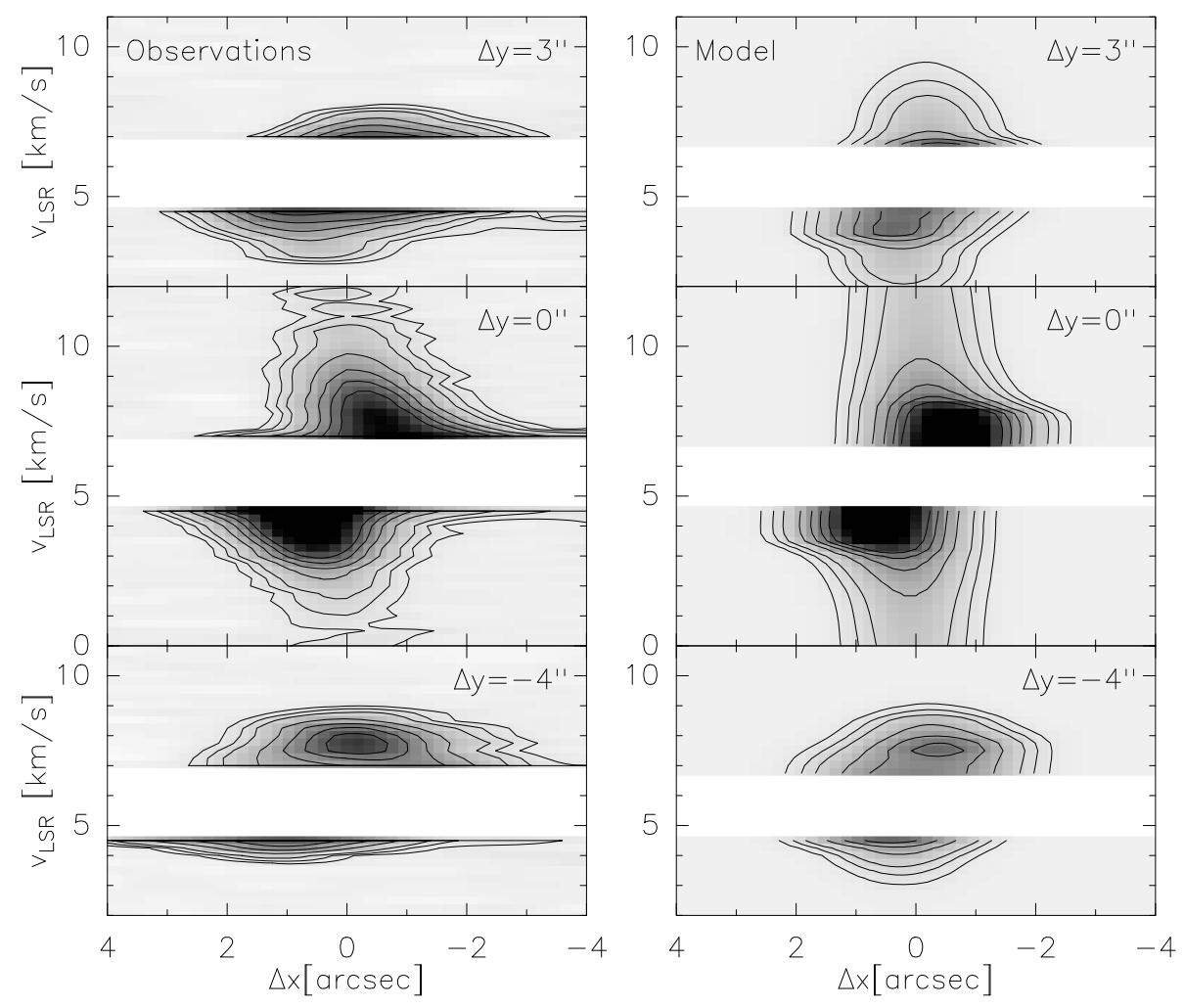

Fig. 5. Position-velocity diagrams of ${ }^{12} \mathrm{CO}(2-1)$ perpendicular to the jet axis. The coordinate along the jet axis $(\Delta y)$ is written in the top right corner of each panel. Contours start at $60 \mathrm{mJy} /$ beam $(3 \sigma)$. Left panel: observations. Right panel: best-fit model.

millimeter wavelengths. In particular, the following parameters were derived: $\alpha_{\rho}=2.2, h_{0}=10 \mathrm{AU}$, and $\alpha_{h}=1.4$. The disk mass remains somewhat uncertain, since the grain properties, and hence the dust opacities, are not well-constrained. Assuming that this young disk (see Sect. 5.1) has a "standard" interstellar gas-to-dust ratio, its total mass would be $\geq 0.1 M_{\odot}$. This mass is consistent with the simple estimate derived directly from the $1.3 \mathrm{~mm}$ continuum flux of $190 \mathrm{mJy}$ and using $\kappa_{1.3 \mathrm{~mm}}=1.0 \mathrm{~cm}^{2} \mathrm{~g}^{-1}$ of dust (Ossenkopf \& Henning 1994), a "standard" gas-to-dust ratio of 100 , and $\left\langle T_{\mathrm{d}}\right\rangle \approx 20 \mathrm{~K}$. The exact value of the disk mass has very little impact on our result, as the $\mathrm{CO}$ emission is optically thick.
The disk was found to have an inner hole of radius $\approx 45 \mathrm{AU}$. Note that the $1.1 \mathrm{~mm}$ continuum image presented in Fig. 1 shows two peaks, which already strongly suggest the presence of an inner gap. The disk modeling also indicates an outer radius of 200 AU.

The adopted disk model was combined with line radiative transfer (LRT) analysis of the $\mathrm{HCO}^{+}(1-0)$ and ${ }^{12} \mathrm{CO}(2-1)$ Keplerian velocity profiles to derive the dynamical mass of the central star(s) and the inclination of the disk, $M_{*}=0.5 \pm 0.1 M_{\odot}$ and $i=85 \pm 4^{\circ}$, respectively. This mass is somewhat higher, but still comparable to the value of $\approx 0.3 M_{\odot}$ derived by Launhardt $\&$ Sargent (2001) from ${ }^{13} \mathrm{CO}(1-0)$ only. 

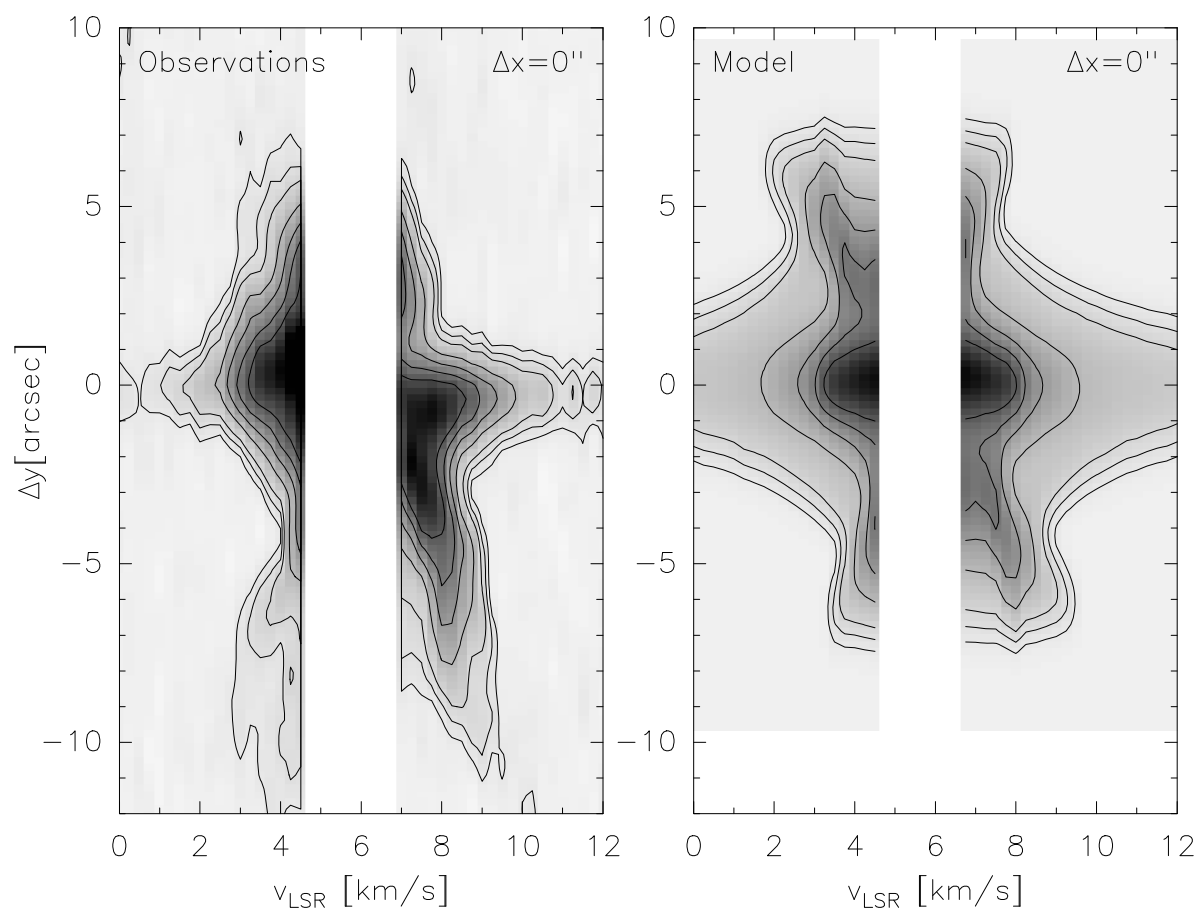

Fig. 6. Position-velocity diagrams of ${ }^{12} \mathrm{CO}(2-1)$ parallel to the jet axis at $\Delta x=0 "$. Contours start at $60 \mathrm{mJy} /$ beam $(3 \sigma)$. Left panel: observations. Right panel: best-fit model.
The ${ }^{12} \mathrm{CO}(2-1)$ emission of this disk was then computed and added to the outflow model described below, to be compared to the observations. The parameters derived for the outflow do not critically depend on the details of the adopted disk model. A much simpler disk model, which we used first, lead to the same conclusions for the outflow.

\subsection{Outflow model}

As there exists no unified physical paradigm for molecular outflows, their prescription still relies on empirical parameterized models adapted to the particular source. To model the CB 26 outflow, we use a conical geometry of maximal size $r_{0}$ (see Fig. 7). To reproduce the shape of the position-velocity diagram along the jet axis (Fig. 6), we assume that the radial outflow velocity is a linear function of the distance from the star: $V(r)=V_{0} \cdot r / r_{0}$. Such "Hubble" law-type velocity fields have been observed in many molecular outflows (e.g., Lada \& Fich 1996), but their origin is not yet well understood (see also Downes \& Cabrit 2003). We further consider a steady-state model: at any radial distance $r$ from the central star, $r^{2} n(r) V(r)=r_{0}^{2} n_{0} V_{0}$, where $V_{0}$ and $n_{0}$ are the radial outflow velocity and density at distance $r_{0}$. The radial $\mathrm{H}_{2}$ density distribution is then given by $n(r)=n_{0} \cdot\left(r / r_{0}\right)^{-3}$. For the distribution of the kinetic gas temperature in the outflow we assume a power law: $T(r)=T_{0}\left(r / r_{0}\right)^{-q}$.

We assume that the outflow is rotating around its polar axis. In principle, one can consider different prescriptions for the radial dependence of the rotation velocity of the outflow. We chose a simple prescription, $V_{\text {rot }} \propto R^{\mu}$, and modeled the following cases: $\mu=1$ (solid body rotation), $\mu=-0.5$ (Keplerian rotation), and $\mu=-1$ (angular momentum conservation). However, the data did not allow us to reliably distinguish between these cases and to further constrain the parameter $\mu$. Therfore, we adopted the physically most plausible scenario in this range. We assume that the rotation velocity is given by $V_{\text {rot }}(R)=V_{\text {kep }}\left(R_{L}\right) \cdot R_{L} / R$, where $R$ is the distance from the outflow axis and $V_{\text {kep }}\left(R_{L}\right)$ is the Keplerian velocity at $R_{L}$ (see Fig. 7 and discussion in Sect. 5.3). This prescription is equivalent to the conservation of the angular momentum along the flow, if all material was ejected from the disk at distance $R_{L}$ with the tangential component of the ejection velocity equal to $V_{\text {kep }}\left(R_{L}\right)$.

Finally, we added a microturbulent velocity of $0.1 \mathrm{~km} \mathrm{~s}^{-1}$, which is a typical value for protoplanetary disks (Guilloteau \& Dutrey 1998) and assumed that CO is not depleted and has a constant abundance of $\mathrm{X}(\mathrm{CO})=7.5 \times 10^{-5}$ (see Sect. 5.3). Synthetic ${ }^{12} \mathrm{CO}(2-1)$ channel maps were produced using the LRT code URAN(IA) (Pavlyuchenkov et al. 2007), taking as input both the outflow and the disk model predictions. The free parameters $V_{0}, R_{L}, n_{0}, T_{0}$, and $q$ of the outflow model were then derived by fitting the synthetic maps to the observations. The computed maps were convolved with a Gaussian beam of $1.5^{\prime \prime}$ and compared with the observed channel maps using the $\chi^{2}$ criterion:

$\chi^{2}=\frac{1}{N \delta I^{\mathrm{obs}}} \sum_{\mathrm{pos}, \mathrm{V}}\left(I_{\mathrm{pos}, \mathrm{V}}^{\mathrm{mod}}-I_{\mathrm{pos}, \mathrm{V}}^{\mathrm{obs}}\right)^{2}$.

Here, $I_{\text {pos, } V}^{\mathrm{mod}}$ and $I_{\mathrm{pos}, \mathrm{V}}^{\mathrm{obs}}$ are the modeled and observed intensities for a given position (pixel) and velocity channel, $\delta I^{\text {obs }}$ is the noise level of the observed intensity in the channel maps, and a summation is made over all positions and velocity channels in the observed map $\left(N=N_{\text {pos }} \times N_{\mathrm{V}}\right)$. Because of the large number of free parameters, we performed the $\chi^{2}$-minimization in several $2 \mathrm{D}$ cuts of the parameter space. This does not guarantee the exact localization of the minimum, but gives a reasonable approximation to it. This approach is justified since our goal was not to derive precise outflow parameters but rather to illustrate that the observed $\mathrm{CO}$ emission can indeed be described by a rotating outflow.

\section{Results}

\subsection{The disk and central star(s)}

Although we have no direct indication of a double star or double jet in CB 26, the large inner hole in the disk of $R_{\text {in }} \sim 45 \mathrm{AU}$ (see 


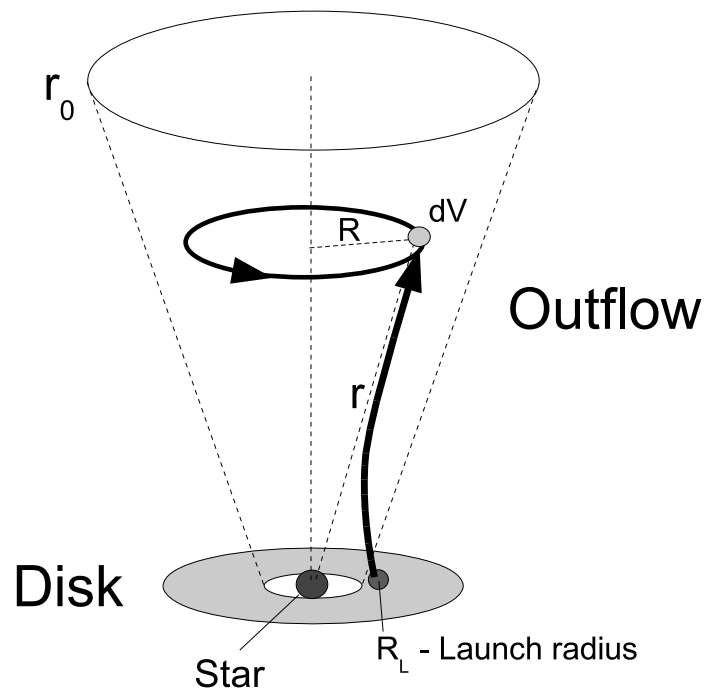

Fig. 7. Schematic sketch of the outflow model. $d V$ is the selected element of the outflow, $r$ and $R$ are the radial and cylindrical coordinates of the selected element, respectively. The element is launched from the disk at radius $R_{L} . r_{0}$ is the length of the outflow (see Sect. 4.2). Matter flow lines (schematic) are shown as solid lines with arrows, while pure geometric connecting lines are dashed.

Sect. 4.1) suggests the presence of a binary system. Assuming that this hole is due to tidal truncation by a binary system with approximately equal masses, the components should have a separation of $a \sim 20$ AU (see Artymowicz \& Lubow 1994). As noted above, the Keplerian profiles of the line emission indicate a total stellar mass of $0.5 \pm 0.1 M_{\odot}$.

Guilloteau et al. (2008) derived very similar properties (stellar mass, size of the central gap) for the HH 30 disk. Combining with the results of the optical jet study of Anglada et al. (2007), they also infer the presence of a binary system with separation $a \sim 15 \mathrm{AU}$, orbital period $P \sim 80 \mathrm{yrs}$, and total mass $\sim 0.5 M_{\odot}$, although the mass ratio remains poorly constrained.

Integrating the spectral energy distribution (SED) of CB 26 yields a total luminosity of $L_{\mathrm{bol}} \geq 0.5 L_{\odot}$ (Stecklum et al. 2004). This can only be a lower limit to the intrinsic luminosity since the edge-on view and bipolar geometry cause an anisotropic radiation field where only a fraction of the NIR photons are scattered into the line of sight. Higher intrinsic stellar luminosities of up to $\sim 2 L_{\odot}$ are consistent with the model (but imply slightly different morphologies of the disk and envelope). Our model corresponds to $L_{\text {bol }}=0.9 L_{\odot}$. Nevertheless, this luminosity range does allow an estimate of the age of the system. Using solar-metalicity PMS tracks of Siess et al. (2000) and a star mass of $0.5 M_{\odot}$, we derive an age of $1 \mathrm{Myr}$, with lower and upper limits of $0.6 \mathrm{Myr}$ and $2 \mathrm{Myr}$, respectively. Assuming two stars of $0.25 M_{\odot}$ each, we derive a similar age range, but the lower limit shifts to $0.1 \mathrm{Myr}$. However, given the well-developed disk and the relatively thin remnant envelope, such a young age does not seem realistic. We can therefore safely constrain the age of CB 26 to be $\approx 1 \mathrm{Myr}$ with an uncertainty of factor 2 .

\subsection{The outflow}

Table 1 summarizes the parameters of the best-fit outflow model. The radial velocity field of the model can be compared to the observations (Fig. 4): it reproduces well the mean radial velocity pattern (red and blue areas) and the S-like shape of the zero offset velocity emission (green area). Furthermore, the computed
Table 1. Parameters of the outflow model for CB 26.

\begin{tabular}{llll}
\hline \hline Parameter & Symbol & fixed & fitted \\
\hline Outflow Size & $r_{0}$ & $1000 \mathrm{AU}$ & - \\
Opening angle & $\alpha$ & $40 \mathrm{deg}$ & - \\
Positional angle & $\mathrm{PA}$ & $30 \mathrm{deg}$ & - \\
Inclination & $i$ & $85 \mathrm{deg}$ & - \\
Microturbulent velocity & $V_{\text {turb }}$ & $0.1 \mathrm{~km} \mathrm{~s}^{-1}$ & - \\
$\mathrm{CO} / \mathrm{H}_{2}$ abundance: & $\mathrm{X}(\mathrm{CO})$ & $7.5 \times 10^{-5}$ & - \\
$\mathrm{H}_{2}$ density at $r_{0}$ & $n_{0}$ & - & $2 \times 10^{4} \mathrm{~cm}^{-3}$ \\
Radial outflow velocity at $r_{0}$ & $V_{0}$ & - & $10 \mathrm{~km} \mathrm{~s}^{-1}$ \\
Temperature at $r_{0}$ & $T_{0}$ & - & $15 \mathrm{~K}$ \\
Temperature index & $q$ & - & 0.5 \\
Rotation velocity at $R=100 \mathrm{AU}$ & $V_{\text {rot }}(100)$ & - & $1.1 \mathrm{~km} \mathrm{~s}^{-1}$ \\
\hline
\end{tabular}

position-velocity diagrams show the same radial velocity shifts across the outflow as the observed data (Fig. 5). Although we can qualitatively reproduce all significant features which are indicative of outflow rotation, the model fit is not perfect. In particular, we cannot reproduce the asymmetry of the outflow along its axis and the exact shape of the position-velocity diagrams. We believe that this is a drawback of our simplified and symmetric model.

The mean $\mathrm{H}_{2}$ density in the outflow at $r_{0}=1000 \mathrm{AU}$ is $2 \times 10^{4} \mathrm{~cm}^{-3}$. The kinetic gas temperature at this radius is $\geq 15 \mathrm{~K}$. The ${ }^{12} \mathrm{CO}(2-1)$ emission is moderately optically thick throughout most of the outflow. The derived radial outflow velocity at $1000 \mathrm{AU}$ of $10 \mathrm{~km} \mathrm{~s}^{-1}$ is comparable to that of HH 30 (Pety et al. 2006), but has a large uncertainty (up to an order of magnitude) because of the uncertain inclination (see below). The rotation velocity is $\sim 1 \mathrm{~km} \mathrm{~s}^{-1}$ at a distance of $100 \mathrm{AU}$ from the ejection axis.

The dynamical timescale of the molecular outflow (ratio of projected length and mean radial velocity, independent of the inclination) is $\tau_{\text {dyn }} \approx 500$ yr. This is much shorter than the estimated age of the source of $\approx 1 \mathrm{Myr}$ (Sect. 5.1). However, the presence of the Herbig-Haro object HH 494 at a much larger distance (Fig. 1 and Stecklum et al. 2004) indicates that the total size, and hence the age, of the outflow is much larger than what we currently see in the ${ }^{12} \mathrm{CO}(2-1)$ line.

The total mass of the visible molecular outflow of $\approx 10^{-3} M_{\odot}$ corresponds to about $1 \%$ of the disk mass. Although it is at the low-energy end, the CB 26 outflow fits well on the observationally established source luminosity - outflow momentum flux relation for protostars presented by Richer et al. (2000).

\subsection{Modeling uncertainties}

Interferometric channel maps of molecular lines from circumstellar disks can be used to constrain the inclination angle and derive the kinematic mass of the central star (Guilloteau \& Dutrey 1998; Simon et al. 2000). However, there is a general degeneracy between disk inclination and star mass. While for faceon configurations the inclination angle can be well-constrained and the star mass remains uncertain, the situation is opposite for edge-on configurations such as CB 26. Using geometrical arguments, we could however constrain the inclination angle of the CB 26 disk to be $\leq 89^{\circ}$ (assuming the radial outflow velocity is $<100 \mathrm{~km} \mathrm{~s}^{-1}$ ) and $>80^{\circ}$ (from the aspect ratio of the $1.3 \mathrm{~mm}$ dust continuum emission). Therefore, we adopted a value of $i=85^{\circ} \pm 4^{\circ}$. In this inclination range, the central star mass is well-constrained by the Keplerian rotation field and we derive $M_{*}=0.5 \pm 0.1 M_{\odot}$. However, the projection factor $1 / \cos i$ has a total uncertainty of one order of magnitude, which leads to 
correspondingly large uncertainties in estimates of outflow energetics and timescales.

Another degeneracy exists between hydrogen density and $\mathrm{CO}$ abundance. This would lead to corresponding uncertainties in the estimates of outflow mass and angular momentum. We cannot directly derive the $\mathrm{CO}$ abundance from our data, but we argue that the mean kinetic gas temperature in the outflow of $\geq 15 \mathrm{~K}$ is high enough to release all CO molecules from the grain mantles into the gas phase (Bisschop et al. 2006) and assume maximum possible $\mathrm{CO}$ abundance.

A more severe uncertainty comes from the fact that the small number of usable velocity channels (due to the effect of the envelope; see Sect. 2 and Figs. 2, 5, and 6) and the moderate SNR of the data did not allow us to well-constrain the radial dependence of the outflow rotation velocity, $V_{\text {rot }}(R)$. The assumption of $V_{\text {rot }} \propto R^{-1}$ was made because it is the physically most plausible profile within the range of profiles that are compatible with the data. However, we also want to stress that the other outflow parameters and conclusions we derive in this paper do not critically depend on the exact radial profile of the outflow rotation velocity.

\section{Discussion}

\subsection{Alternative explanations for the velocity field of the outflow}

The previous sections presented the model of a rotating outflow, that was successfully fitted to the data. However, we also considered a number of other configurations that could possibly lead to the observed peculiar velocity field of the CB 26 outflow:

1. molecular clouds which intersect with the line of sight and mimic a rotation-like velocity pattern;

2. two mis-aligned (non-rotating) outflows that are not spatially resolved, and

3. a precessing (non-rotating) jet/outflow.

Given the relatively isolated location of the small globule CB 26 (see Fig. 1), the clear jet-like bipolar morphology of the $\mathrm{CO}$ emission, and the single-component line shapes (see Fig. 2), scenario (1) would require a very special spatial and kinematic configuration of the environment and seems extremely unlikely. The alignment of $\mathrm{HH} 494\left(\mathrm{PA}=145^{\circ}\right)$ with the axis of the $\mathrm{CO}$ outflow $\left(\mathrm{PA}=147 \pm 3^{\circ}\right.$ ) also argues against this scenario (see Fig. 1).

We tried to model two (spatially unresolved) outflows that are slightly misaligned with respect to the line of sight (scenario 2). The suspected binarity of CB 26 could make this scenario appealing. However, until now, only a very few close binary systems are known to drive two jets; e.g., there is evidence that the quadrupolar outflow associated with $\mathrm{HH} 111$ is driven by a close binary (separation $<40 \mathrm{AU}$ in projection; Rodríguez et al. 2008). Two outflows from the same central core (the so-called quadrupolar outflows) are usually observed only from sources with much larger separations (e.g., a few thousand AU in HH 288 or CG 30; Gueth et al. 2001; Chen et al. 2008). Still, we have modeled this configuration and found that it can also produce an effective velocity gradient across the map of the two combined flows. However, reproducing the S-shape of the zero-velocity zone in the 1st moment map (green area in Fig. 4) requires finetuning of both the relative orientation and the strength the two flows. The most significant differences to a rotating configuration occur near the driving sources and at low radial velocities. Unfortunately, both the dominating disk emission at the center and the missing low-velocity channels due to masking by the non-recoverable envelope emission prevent us from ultimately confirming or disproving this scenario as a possible origin of the peculiar velocity field of CB 26 . Note, however, that the agreement between the velocity gradient directions of disk and outflow would be a coincidence in this scenario.

Entrainment by an unseen precessing jet (scenario 3 ) could possibly also mimic a similar radial velocity field when the outflow traces about 0.25 to 0.5 of the precession period. In this case, we would not observe rotation, but see gas that is accelerated along different directions at different times. However, longitudinal gradients would dominate the radial velocity field over lateral ones. Since simulating this scenario would require a nonstationary model, we have only considered it as a thought experiment. Assuming that the precession of the jet axis is driven by tidal interactions between the circumstellar disk from which the jet is launched and a companion star on a non-coplanar orbit, the shortest possible precession period in CB 26 would be of the order of $2000 \mathrm{yr}$ (Terquem 1998; Anglada et al. 2007, see their Eq. (14)). Here we assumed equal companion masses, small inclination angles, the maximum ratio between the circumstellar disk radius and a companion separation of 0.3 , and an orbital period of $\approx 100 \mathrm{yr}$ (Sect. 5.1). Comparing this to the dynamical timescale of the molecular outflow of $500 \mathrm{yr}$ (Sect. 5.2) leads to the conclusion that at least the timescales are such that this scenario cannot be excluded from being at work for CB 26 . However, $t \mathrm{t}$ would be worthwhile to test the precession scenario with an appropriate model.

\subsection{A rotating outflow - what distinguishes CB26 from other sources?}

Assuming that the velocity pattern of the CB 26 outflow does result from a rotation of the outflowing material about its ejection axis, the data presented in this paper would be the first observational signature of this phenomenon.

If the angular momentum transfer from the disk by an (unseen) rotating jet drives the rotation of the molecular outflow, the question that arises is why such a signature is so prominent in CB 26 while it has not been observed in other sources? Detecting the projected velocity gradients requires a nearly edge-on configuration, which is the case of CB 26 but also of other sources that have been studied. Some other specificities of CB 26 must therefore be involved.

The comparison between the CB 26 and HH 30 (Pety et al. 2006; Guilloteau et al. 2008) cases illustrates that difficulty. In many aspects, these two sources are very similar: both systems are seen almost edge-on, with an inclination greater than $\sim 84^{\circ}$; the disks are in Keplerian rotation around a $\sim 0.5 M_{\odot}$ system and have an $\sim 40$ AU inner gap; and both sources drive compact molecular outflows. Binarity seems the most simple explanation for both the $\mathrm{mm}$ and optical appearance of HH 30 (Anglada et al. 2007; Guilloteau et al. 2008), while the binarity status of CB 26 is still speculative. If CB 26 is indeed a binary system, it is likely to have similar characteristics to those of $\mathrm{HH} 30$, as the masses of the central star(s) and the sizes of the cavities are similar. As for the $\mathrm{CO}$ outflow, the $\mathrm{HH} 30$ flow has a somewhat wider opening angle $\left(60^{\circ}\right)$ than $\mathrm{CB} 26\left(40^{\circ}\right.$, see Table 1$)$ and presents only one lobe (which remains a puzzling feature, probably related to the environment of the protostar rather than to an intrinsic property of the ejection mechanism). More importantly, and despite a detailed analysis of the $\mathrm{CO}$ emission, the HH 30 outflow does not show any sign of rotation (Pety et al. 2006). If the velocity pattern observed in CB 26 is indeed rotation, this raises the 
Table 2. Derived parameters of outflow and disk.

\begin{tabular}{lll}
\hline \hline Parameter & Symbol & Value \\
\hline Total outflow mass & $M_{\mathrm{CO}}$ & $1 \times 10^{-3} M_{\odot}$ \\
Mass flux & $\dot{M}_{\mathrm{CO}}$ & $2 \times 10^{-7} M_{\odot} \mathrm{yr}^{-1}$ \\
Total outflow momentum & $P_{\mathrm{CO}}$ & $1 \times 10^{-2} M_{\odot} \mathrm{km} \mathrm{s}^{-1}$ \\
Momentum flux & $\dot{P}_{\mathrm{CO}}$ & $2 \times 10^{-6} M_{\odot} \mathrm{km} \mathrm{s}^{-1} \mathrm{yr}^{-1}$ \\
Total angular momentum & $J_{\mathrm{CO}}$ & $1.5 \times 10^{7} M_{\odot} \mathrm{km}^{2} \mathrm{~s}^{-1}$ \\
Angular momentum flux & $\dot{J}_{\mathrm{CO}}$ & $3 \times 10^{3} M_{\odot} \mathrm{km}^{2} \mathrm{~s}^{-1} \mathrm{yr}^{-1}$ \\
Specific angular momentum & $j_{\mathrm{CO}}$ & $1.5 \times 10^{10} \mathrm{~km}^{2} \mathrm{~s}^{-1}$ \\
Disk mass* & $M_{\text {disk }}$ & $1 \times 10^{-1} M_{\odot}$ \\
Disk angular momentum & $J_{\text {disk }}$ & $3 \times 10^{9} M_{\odot} \mathrm{km}^{2} \mathrm{~s}^{-1}$ \\
Disk specific angular momentum & $j_{\text {disk }}$ & $3 \times 10^{10} \mathrm{~km}^{2} \mathrm{~s}^{-1}$ \\
\hline
\end{tabular}

* The disk mass is derived from the disk model of Sautter et al. (in prep.).

question of why rotation is not observed in a similar object like HH 30? The most significant difference between the two sources is their age: CB 26 is likely to be younger, since it has a more prominent envelope. However, there are no clear prescriptions or predictions of the evolution of the jet/outflow rotation with time.

More generally, we note that the angular momentum transfer from the central sources to the outflowing gas may be quite complex in a binary system - as suspected in CB 26. For example, one could speculate on the presence of two distinct parallel jets, creating one single outflow, but "stiring" the entrained gas and dispersing orbital angular momentum from the system (cf. Masciadri \& Raga 2002).

\subsection{The relation between disk and outflow}

If we make the two simplifying assumptions that the outflow mass flux is constant over time and that the outflow is fed directly by the disk, it would take $\approx 0.5 \mathrm{Myr}$ to dissipate the disk (see Table 2). This compares well with the age of the system and, within the uncertainty range, with the typical dispersal time of circumstellar disks around young stars (Haisch et al. 2001; Cieza et al. 2007). We also note that this mass outflow rate is comparable to typical accretion rates for young $\mathrm{T}$ Tauri stars (Hartigan et al. 1995; Gullbring et al. 1998). Assuming that the outflow indeed rotates, the average specific angular momentum of the outflow gas is somewhat lower than or comparable to that of the disk material. If we make the same assumptions for the angular momentum dispersal as above, we derive a timescale of $\approx 1 \mathrm{Myr}$. The good agreement between both balance and timescale estimates supports the hypothesis that the CB 26 outflow is indeed rotating and dispersing angular momentum from the system. It also shows that in CB 26 the outflow plays a significant role in dispersing the disk, and thus terminating the conditions for planet formation.

The rotation of the outflowing gas also can be used to place constraints on the size of the ejecting region in the disk, assuming we are observing gas that has been directly ejected from the disk, and not envelope material that has been accelerated by an underlying (unseen) jet. Using the simple assumptions that the outflow material is launched from a single point on the Keplerian disk and that angular momentum is conserved, i.e.,
$R \cdot V_{\text {rot }}(R)=R_{L} \cdot V_{\text {kep }}\left(R_{L}\right)$, we derive from our $\chi^{2}$-fitting routine $R_{\mathrm{L}} \approx 30 \mathrm{AU}$. This is consistent with the simple estimation

$R_{L}=\frac{\left(V_{\mathrm{rot}} R\right)^{2}}{G M_{*}}$

which yields $R_{\mathrm{L}} \approx 27 \mathrm{AU}$ when we assume $M_{*}=0.5 M_{\odot}, R=$ $100 \mathrm{AU}$, and $V_{\text {rot }}=1.1 \mathrm{~km} \mathrm{~s}^{-1}$ (see Table 1 ).

One can also use magneto-centrifugal wind models (see review by Shu et al. 2000) to constrain the outflow launch region. In this theory, the magnetic field plays an essential role in extracting the energy and angular momentum from the disk and redirecting them to the outflow. Anderson et al. (2003) have shown that there is a useful invariant that is conserved along a magnetic field line and that can be used to determine the radius $R_{\mathrm{ML}}$ at which the material was ejected. Pety et al. (2006) have shown that this relation can be written as

$R_{\mathrm{ML}}=R\left(2 \frac{V_{\mathrm{kep}} V_{\mathrm{rot}}}{V^{2}}\right)^{2 / 3}$,

where $R$ is the distance from the outflow axis to the considered volume element, $V_{\text {kep }}=\sqrt{G M_{*} / R}$ is the Keplerian velocity at this position, and $V_{\text {rot }}$ and $V$ are the measured rotation and radial outflow velocities at that position. However, the Hubble lawtype radial outflow velocity field (see Sect. 4.2) implies that the launching radius derived with this equation depends on the distance of the measurement point from the star. For Eq. (4) to be applicable, this measurement point should far away from the star (Anderson et al. 2003). On the other hand, the Hubble law describes the observed radial outflow velocity field of CB 26 reasonably well at small and intermediate distances from the star, while the outflow velocity appears to be more constant at larger distances (see Fig. 6). If we therefore use $V=5 \mathrm{~km} \mathrm{~s}^{-1}$ at $r=500 \mathrm{AU}$ (see Table 1 and Sect. 4.2), and further assume $M_{*}=0.5 M_{\odot}, R=100 \mathrm{AU}$, and $V_{\text {rot }}=1.1 \mathrm{~km} \mathrm{~s}^{-1}$, we obtain $R_{\mathrm{ML}} \approx 32 \mathrm{AU}$. This value is remarkably close to the values obtained from the simple estimates above. Using $V=10 \mathrm{~km} \mathrm{~s}^{-1}$ at $r=1000 \mathrm{AU}$ leads to $R_{\mathrm{ML}} \approx 13 \mathrm{AU}$, which can be considered a lower limit to $R_{\mathrm{ML}}$. However, as all these estimates are based on a number of unproven assumptions, we do not use them to draw conclusions on the physical launch mechanism.

\section{Summary and conclusions}

We presented new millimeter-interferometric observations of the young edge-on T Tauri star - disk system in the isolated Bok globule $\mathrm{CB} 26$. Our ${ }^{12} \mathrm{CO}(2-1)$ line data at 1 ..5 spatial and $0.25 \mathrm{~km} \mathrm{~s}^{-1}$ velocity resolution reveal a previously undiscovered collimated bipolar molecular outflow of total length $\approx 2000 \mathrm{AU}$, escaping perpendicular to the plane of the well-resolved circumstellar disk in Keplerian rotation. The disk has an inner hole of radius $\approx 45 \mathrm{AU}$, indicating the possible presence of a binary star system with separation $\approx 20$ AU. The dynamic mass and age of the central obscured $\operatorname{star}(\mathrm{s})$ is $0.5 \pm 0.1 M_{\odot}$ and $\approx 1 \mathrm{Myr}$, respectively.

The outflow shows very clear and consistent kinematic signatures that suggest it is rotating about its polar axis. We use an empirical parameterized outflow model combined with 2-D line radiative transfer calculations and $\chi^{2}$-minimization in parameter space to derive a best-fit model and compute parameters of the outflow. We could show that the data are consistent with outflow rotation. This hypothesis is supported by the fact that the disk and outflow seem to be co-rotating and fit together energetically. 
However, we could not exclude jet precession or two misaligned flows as possible origins of the observed peculiar velocity field.

Assuming that the CB 26 outflow is rotating, we computed and compared mass, mass flux, angular momenta, and angular momentum flux of disk and outflow and derived disk dispersal timescales of $0.5 \ldots 1 \mathrm{Myr}$. Although our simplifying assumptions and the model uncertainties do not allow us to draw more precise conclusions about the outflow formation and disk dispersal mechanisms, this result confirms the typical disk dispersal timescale derived by statistical arguments. It also shows that the CB 26 outflow must play a significant role in dispersing the disk.

Testing the proposed scenarios that could explain the peculiar radial velocity field of the CB 26 molecular outflow will most likely require a combination of the radiative transfer calculations and hydrodynamic models of jet formation and entrainment in multiple sources.

Acknowledgements. We acknowledge the Plateau de Bure and Grenoble IRAM staff for their help during the observations and data reduction. This work was supported by the "Action sur Projets Physique Chimie du Milieu Interstellaire" (PCMI) from INSU/CNRS. J. Sauter and S. Wolf provided us with their preliminary results on the disk modeling and we benefitted from many fruitful discussions with them. We also want to thank K. Schuster and C. Fendt for helpful discussions. B. Stecklum kindly provided the color composite image used for the overview figure. We are grateful to G. Anglada for critically reading the manuscript and providing us with constructive comments that helped to improve the correctness and clarity of this paper.

\section{References}

Anderson, J. M., Li, Z.-Y., Krasnopolsky, R., \& Blandford, R. D. 2003, ApJ, 590, L107

Anglada, G., López, R., Estalella, R., et al. 2007, AJ, 133, 2799

Artymowicz, P., \& Lubow, S. H. 1994, ApJ, 421, 651

Bacciotti, F., Ray, T. P., Mundt, R., Eislöffel, J., \& Solf, J. 2002, ApJ, 576, 222

Bacciotti, F., Ray, T. P., Eislöffel, J., et al. 2003, Ap\&SS, 287, 3

Bisschop, S. E., Fraser, H. J., Öberg, K. I., van Dishoeck, E. F., \& Schlemmer, S. 2006, A\&A, 449, 1297
Chen, X., Bourke, T. L., Launhardt, R., \& Henning, T. 2008, ApJ, 686, L107 Chrysostomou, A., Bacciotti, F., Nisini, B., et al. 2008, A\&A, 482, 575 Cieza, L., Padgett, D. L., Stapelfeldt, K. R., et al. 2007, ApJ, 667, 308 Codella, C., Cabrit, S., Gueth, F., et al. 2007, A\&A, 462, L53

Coffey, D., Bacciotti, F., Woitas, J., Ray, T. P., \& Eislöffel, J. 2004, ApJ, 604, 758

Coffey, D., Bacciotti, F., Ray, T. P., Eislöffel, J., \& Woitas, J. 2007, ApJ, 663, 350

Downes, T. P., \& Cabrit, S. 2003, A\&A, 403, 135

Dutrey, A., Henning, T., Guilloteau, S., et al. 2007, A\&A, 464, 615 Gueth, F., Schilke, P., \& McCaughrean, M. J. 2001, A\&A, 375, 1018

Guilloteau, S., \& Dutrey, A. 1998, A\&A, 339, 467

Guilloteau, S., Dutrey, A., Pety, J., \& Gueth, F. 2008, A\&A, 478, L31

Gullbring, E., Hartmann, L., Briceno, C., \& Calvet, N. 1998, ApJ, 492, 323

Haisch, Jr., K. E., Lada, E. A., \& Lada, C. J. 2001, AJ, 121, 2065

Hartigan, P., Edwards, S., \& Ghandour, L. 1995, ApJ, 452, 736

Henning, T., Wolf, S., Launhardt, R., \& Waters, R. 2001, ApJ, 561, 871

Lada, C. J., \& Fich, M. 1996, ApJ, 459, 638

Launhardt, R., \& Henning, T. 1997, A\&A, 326, 329

Launhardt, R., \& Sargent, A. I. 2001, ApJ, 562, L173

Lee, C.-F., Ho, P. T. P., Palau, A., et al. 2007, ApJ, 670, 1188

Lee, C.-F., Ho, P. T. P., Bourke, T. L., et al. 2008, ApJ, 685, 1026

Lenzen, R., Bizenberger, P., Salm, N., \& Storz, C. 1998, in Infrared Astronomical Instrumentation, ed. A. M. Fowler, Proc. SPIE, 3354, 493

Masciadri, E., \& Raga, A. C. 2002, ApJ, 568, 733

Ossenkopf, V., \& Henning, T. 1994, A\&A, 291, 943

Pavlyuchenkov, Y., Semenov, D., Henning, T., et al. 2007, ApJ, 669, 1262

Pety, J., Gueth, F., Guilloteau, S., \& Dutrey, A. 2006, A\&A, 458, 841

Ray, T., Dougados, C., Bacciotti, F., Eislöffel, J., \& Chrysostomou, A. 2007, in Protostars and Planets V, ed. B. Reipurth, D. Jewitt, \& K. Keil, 231

Richer, J. S., Shepherd, D. S., Cabrit, S., Bachiller, R., \& Churchwell, E. 2000, Protostars and Planets V, 867

Rodríguez, L. F., Torrelles, J. M., Anglada, G., \& Reipurth, B. 2008, AJ, 136, 1852

Shu, F. H., Najita, J. R., Shang, H., \& Li, Z.-Y. 2000, Protostars and Planets IV, 789

Siess, L., Dufour, E., \& Forestini, M. 2000, A\&A, 358, 593

Simon, M., Dutrey, A., \& Guilloteau, S. 2000, ApJ, 545, 1034

Stecklum, B., Launhardt, R., Fischer, O., et al. 2004, ApJ, 617, 418

Terquem, C. E. J. M. L. J. 1998, ApJ, 509, 819

Woitas, J., Bacciotti, F., Ray, T. P., et al. 2005, A\&A, 432, 149

Wolf, S., Launhardt, R., \& Henning, T. 2003, ApJ, 592, 233 